\title{
Electromigration driven failures on miniature silver fuses at the Large Hadron Collider
}

\author{
Nikolaos Trikoupis ${ }^{1}$ \\ CERN-TE/CRG \\ 1211 Geneva 23, Switzerland \\ E-mail: n.trikoupisecern.ch \\ Juan Casas \\ CERN-TE/CRG \\ 1211 Geneva 23, Switzerland \\ E-mail: juan.casas-cubillosecern.ch
}

\section{Ana Teresa Perez Fontenla}

CERN-EN/MME

1211 Geneva 23, Switzerland

E-mail: ana.teresa.perez.fontenla@cern.ch

Spurious faults were observed on the miniature silver fuses of electronic cards used for the cryogenics instrumentation in the LHC (Large Hadron Collider) accelerator at CERN. By applying analytical tools and techniques such as Scanning Electron Microscopy, spectrometry and Weibull reliability calculations and by the knowledge of operating temperatures and operational time of each unit, the origin of the problem has now been understood and can be attributed to electromigration. The selected fuse was operated at moderate temperature and load conditions and was considered as a "lifetime" component. However, it turned out to have a smaller than expected MTTF with failures following a Weibull distribution of $\beta=3.91$ and $\eta=2323$. The literature describes extensively the effects of electromigration, but there are only limited references referring to the impact of this phenomenon on miniature silver fuses for electronic circuits.

Topical Workshop on Electronics for Particle Physics

11 - 14 September 2017

Santa Cruz, California

\section{${ }^{1}$ Speaker}




\section{Introduction}

In the LHC accelerator, the cryogenics instrumentation infrastructure [1] uses fuse-protected high-voltage temperature transducer cards with galvanic insulation to measure the temperatures of the LHC "current leads" [2]. During the periods 2008-2010 [3] and 2014-2016, spurious faults were observed on the miniature silver fuses of those cards requiring in-situ interventions in the LHC tunnel. Following an increasing number of events, and due to the importance of those instrumentation channels for the LHC operation, a study was launched to understand the underlying failing mechanism.

\section{Temperature transducer cards and spurious fuse failures}

The temperatures of the LHC current leads are measured by 1202 temperature cards installed at 19 different LHC shielded locations. The operating current of those cards varies between card families: 1062 cards operate at a current of $500 \mathrm{~mA}$ and 140 cards at below $300 \mathrm{~mA}$. The cards were initially protected by a miniature fuse rated at $1 \mathrm{~A}$ (Schurter type MSF125), based on a 40 um thin silver filament, operating well below the recommended load factor of $70 \%$ as per standard UL 248-14.

During the period 2008-2010, fuse failures started appearing at three LHC locations (total population of 150 cards) as early as 445 days after the installation of their corresponding cards. After 17 such failures, the fuses were replaced by others of the same series offering an increased rated protection of $1.25 \mathrm{~A}$. No further failures were reported in the next 4 years.

During the LHC long shutdown 1 (LS1) that started on February 2013, 764 out of the 1062 cards were upgraded to a new card version consuming less than $300 \mathrm{~mA}$ and only 298 of the original "failing type" remained in service. However, during 2014, new fuse failures occurred for the "failing type" cards and 12 such failures were recorded until 2016.

\section{Problem investigation}

\subsection{Weibull reliability analysis}

The statistical Weibull reliability analysis [4] can be used to correlate failures, occurring over a period of time, to either "early infant mortality", random failures or "wear out failures". This statistical tool requires information for each unit on its cumulative operational time without failure.

In order to apply this tool on the failures of the two periods, it was necessary to extract data from existing databases taking into account possible exchange of cards for repairs, and the periods during which the cards were unpowered. This task was not trivial taking into consideration that the cards were installed 10 years ago.

The results of the Weibull reliability analysis are shown in Figure 1. In those graphs, the failures are plotted on a logarithmic time scale $\mathrm{x}$-axis as per cumulative operating time until failure and on y-axis as per cumulative unavailability of their population. An alignment of the points indicate a common failure mode described by a Weibull factor $\beta$. The MTTF (Mean Time To Failure) is the point where the fitted line has an unavailability of $63.2 \%$.

In the graphs of Figure 1, the calculated Weibull factors of $\beta>>1$ indicate strong end-oflife wear-out failures. When comparing the periods 2008-2010 and 2014-2016 for the same LHC locations, it can be understood that the increase of the fuse rating from $1 \mathrm{~A}$ to $1.25 \mathrm{~A}$ led to an increase of the MTTF from 1687 to 2323 days. This increase could be explained in two ways when considering the decreased current density through the fusing filament of the larger $1.25 \mathrm{~A}$ fuses. First, according to the Arrhenius formula [5], the MTTF would increase by $40 \%$ due to the reduced operating temperature of the filament by $4.5^{\circ} \mathrm{C}$ (as measured experimentally). Secondly, according to Black's equation on electromigration, see Chapter 3.3.1, such an increase would also be expected. 

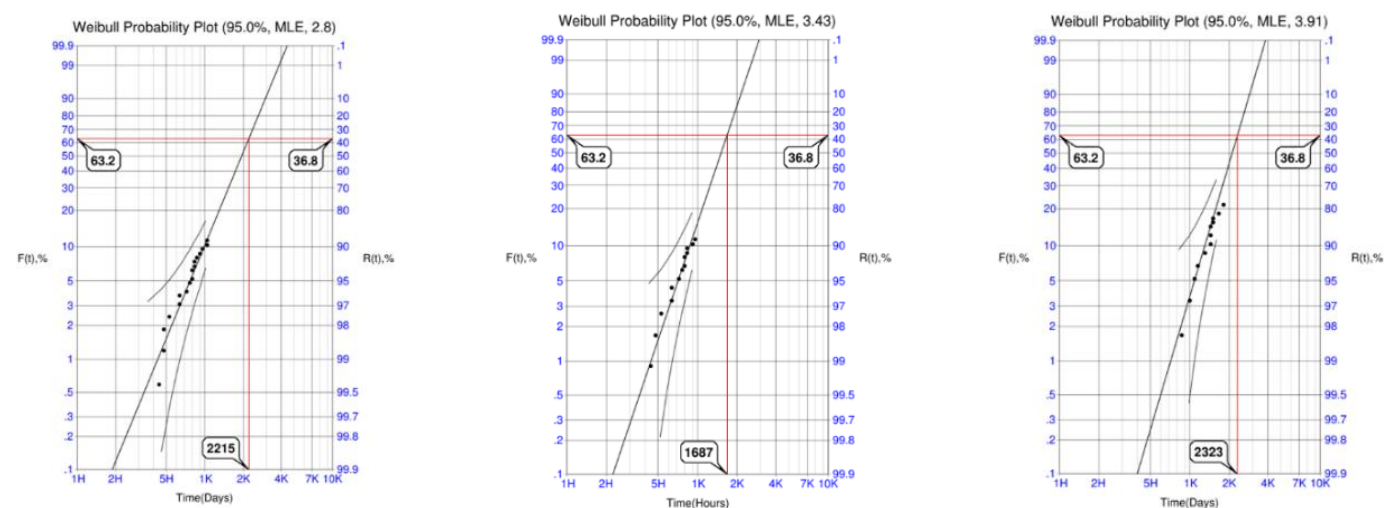

Figure 1: Weibull distributions. The graphs show the MTTF horizontal axis, and the 95\% MLE (Maximum Likelihood Estimation) of the Weibull $\beta$ factor. Left: Period 2008-2010, 17 failures out of 150 cards at 3 LHC locations. Middle: Period 2008-2010: 13 failures out of 102 cards for the same 2 LHC locations as period 2014-2016. Right: Period 2014-2016: 12 failures out of 46 cards at 2 LHC locations.

After communication with the manufacturer, it was confirmed that this device had a "basic MTTF of 125,000 hrs"; a number provided "as is" without reference to current load or operating temperature conditions.

The MTTF values of the current study (55'752 hours), as derived from the Weibull analysis, are well in accordance with another study [6] on similar miniature silver fuses of $40 \mathrm{um}$ fusing filament (75'143 hours).

\subsection{Correlation on failures with operating temperatures}

The LHC locations where the failures appeared are among the warmest. At those locations, the cards operate at temperatures of up to $60{ }^{\circ} \mathrm{C}$; this is because of the tight gaps between the crates and the chosen method of natural convection for air-cooling. In addition, the failures were located mostly at the warmer top part of the double level crates indicating a possible correlation with the operating temperatures.

\subsection{Scanning Electron Microscopy and Energy-Dispersive X-ray Spectroscopy}

Two samples, an unused reference fuse and a fuse that failed in the LHC, were used in a Scanning Electron Microscopy (SEM) and Energy-Dispersive X-ray Spectroscopy (EDS) study as shown in Figure 2. The specimens were coated with a thin layer of gold to create a conductive layer on the sample surface that inhibits charging. This is reflected in the EDS spectrum. The filament of the failed fuse includes distinct regions of material voids and depositions, an indication of electromigration [7]. The EDS analysis shows no differences in the chemical composition between the two samples proving that there were no chemical pollution or corrosion effects.
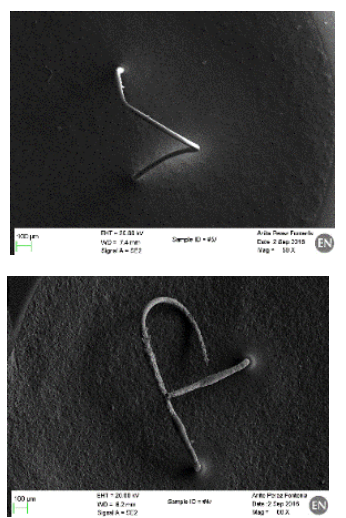
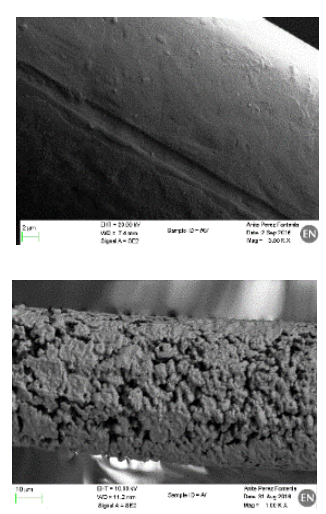
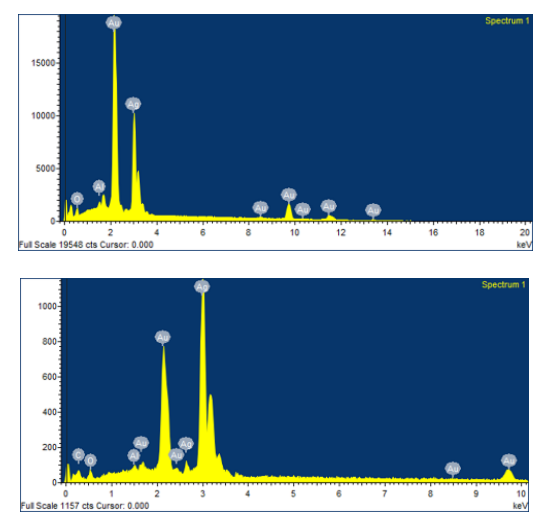

Figure 2: SEM and EDS images. Top row images refer to an unused fuse. Top middle: A close-up of the fuse silver wire with a diameter of $40 \mathrm{um}$. Top right: EDS at a range $0-20 \mathrm{keV}$. Bottom row images refer to 
an LHC failed fuse. Bottom left: Distinct regions of voids and material accumulation. Bottom middle: Appearance of voids in the silver wire. Bottom right: EDS at a range of 0-10 keV.

\subsubsection{Electromigration}

The SEM images revealed effects identical to electromigration. Electromigration is the transport of material due to the momentum transfer between conducting electrons and diffusing metal atoms. The dislocated atoms may form voids at their original location and depositions at their final location. The MTTF of failures due to electromigration can be calculated by Black's equation:

$$
\operatorname{MTTF}=\left(\mathrm{A} / \mathrm{J}^{\mathrm{n}}\right)^{*} \mathrm{e}^{\mathrm{E} / \mathrm{kT}} \quad \text { Black's equation on electromigration [8] }
$$

where $\mathrm{A}$ is a material constant, $\mathrm{J}$ is the current density, Ea is the activation energy, $\mathrm{k}$ is the Boltzmann constant, $\mathrm{T}$ is the temperature and $\mathrm{n}$ a scaling factor.

Bibliography studies have shown that pure silver is highly susceptible to electromigration due to its low activation energy and high mobility of its grain boundaries. Other metals such as aluminium and copper behave in a similar way. One of the methods to reduce the effects of electromigration is the use of alloys, which tend to increase the activation energy and reduce the mobility of the grain boundaries [7], [9].

\subsection{Laboratory stressed samples}

Some fuses of the failing type (1.25 A) were operated under various conditions in the laboratory and they underwent SEM analysis in order to visualize the different types of stress like sudden overcurrent or operation close to maximum tolerable limits. Figure 3 shows three such examples.
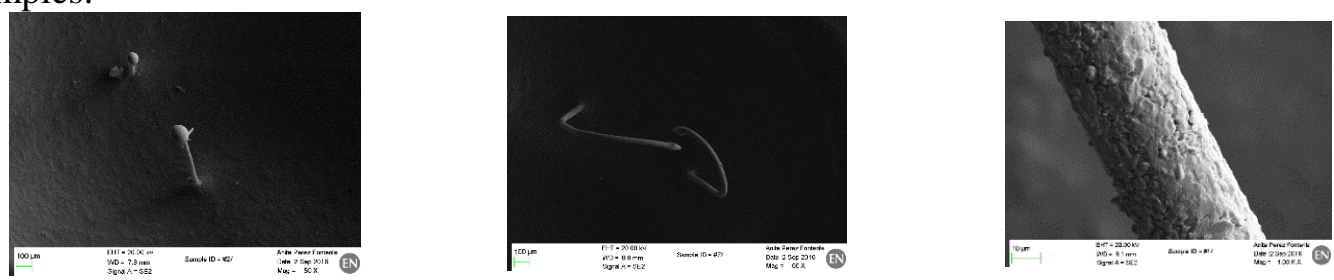

Figure 3: SEM images of laboratory stressed samples. Left: Failure due to overcurrent. Middle: Failure after $4320 \mathrm{ON} / \mathrm{OFF}$ cycles at $1 \mathrm{~A}$ and 576 cycles at $1.35 \mathrm{~A}$ (one cycle per second). Right: A non-failed fuse after operating at $1.25 \mathrm{~A}$ for 120 hours and at $1.35 \mathrm{~A}$ for 16 hours showing material transport phenomena.

\subsection{Accelerated lifetime tests}

The typical resistance of an unused 1.25 A fuse is $\sim 82 \mathrm{mOhm}$. Six fuses were removed from the LHC after operating for $\sim 2000$ days without a failure and their resistance was measured to be in the range of 87 to $97 \mathrm{Ohm}$; these abnormally high values may indicate an aging effect. A highly accelerated lifetime test was then executed on unused fuses in an effort to reproduce the resistive measurements of the LHC fuses and to develop a method of fault prediction.

Four unused fuses of the original failing type and other fuse candidates were tested. Due to the limited time, the accelerated tests were run at high temperatures of $150{ }^{\circ} \mathrm{C}$ and $175^{\circ} \mathrm{C}$ to achieve high acceleration factors as per the Arrhenius model. Those elevated temperatures highly exceed the maximum operating conditions of the fuses. For operation at $175{ }^{\circ} \mathrm{C}$, some of the failures were attributed to material damage (e.g. partial melting of the fuse case). For the runs at $150{ }^{\circ} \mathrm{C}$, no such failures were observed. The fuse filament resistances after the test were significantly higher (Table 1) but did not match those of the six fuses removed from the LHC.

\begin{tabular}{|c|c|c|c|c|}
\hline Fuse & Rating & Load & Conditions & Measurements \\
\hline$\# 1$ & $1.25 \mathrm{~A}$ & $50 \%$ & 1 day $@ 125^{\circ} \mathrm{C}, 2$ days $@ 175^{\circ} \mathrm{C}$ and 15 days $@ 150^{\circ} \mathrm{C}$ & $\begin{array}{l}84.4 \text { mOhm @ 0d, 94.8 mOhm @ 7d, 93.8 mOhm @ 10d, } \\
100.6 \text { mOhm @ 15d, 270 mOhm @ 18d. }\end{array}$ \\
\hline \#2 & $1.25 \mathrm{~A}$ & $80 \%$ & 14 days @ $150^{\circ} \mathrm{C}$ & $\begin{array}{l}83.1 \text { mOhm @ 0d, 85.9 mOhm @3d, 91.2 mOhm @ 8d, } \\
91.3 \text { mOhm @ 11d, 90.9 mOhm @ 14d. }\end{array}$ \\
\hline$\# 3$ & $1 \mathrm{~A}$ & $80 \%$ & 1 day @ $125^{\circ} \mathrm{C}$ and 2 days @ $175^{\circ} \mathrm{C}$ & 112.3 mOhm @ 0d, found failed on day 3. \\
\hline \#4 & $1 \mathrm{~A}$ & $80 \%$ & 18 days @ $150^{\circ} \mathrm{C}$ & $\begin{array}{l}\text { 112 mOhm @ 0d, } 116.4 \text { mOhm @ 4d, } 124.8 @ 7 d, \\
129.7 @ 12 d, 137.9 @ 15 d, \text { failed found on day } 18 .\end{array}$ \\
\hline
\end{tabular}

Table 1: Resistance measurements on accelerated tested fuse samples. 
A predictive formula based on resistive measurements after a highly accelerated test could not be derived, as the aging and electromigration effects develop, most likely, at different rates at those very high temperatures.

\section{Consolidation Year-End Shutdown 2016-2017}

During the LHC year-end shutdown of 2016-2017, the fuses of all insulating temperature cards were replaced by a new type of fuse with a higher stated reliability as per information provided by the manufacturer (Littelfuse 027201.5). Due to limited time, the new fuses were not tested under SEM/EDS to reveal their composition but such a test will be performed. In certain cases, the electronic cabinet separation was increased to improve the natural convection cooling resulting in a lower fuse temperature. Cooling fans were installed at some racks exhibiting higher temperatures values; a temperature reduction of $20^{\circ} \mathrm{C}$ was achieved on the top of one rack.

\section{Summary}

A family of electronic cards was experiencing failures in their miniature silver fuses due to electromigration. The risk of such failures is not sufficiently documented in the bibliography. The datasheets of miniature fuses do not typically provide MTTF values and references for reliability prediction of electronic equipment, such as the MIL-HDBK-217F [10], would result in calculated MTTF values in the range of millions of hours.

Statistical Weibull reliability analysis provided strong indications of the wear-out failure mechanism and the SEM analysis was proven a reliable and effective method of assessing the status of such fuses.

\section{References}

[1] Casas-Cubillos, J., G. Fernandez Penacoba, and M. A. Rodríguez-Ruiz. "The radiation tolerant electronics for the LHC cryogenic controls: Basic design and first operational experience." (2008)

[2] Ballarino, Amalia. "HTS current leads for the LHC magnet powering system." Physica C: Superconductivity 372 (2002): 1413-1418.

[3] Balle, Christoph, Juan Casas, and Nicolas Vauthier. "Analysis of the failures and corrective actions for the LHC cryogenics radiation tolerant electronics and its field instruments." AIP Conference Proceedings. Vol. 1573. No. 1. AIP, 2014.

[4] Abernethy, R. B. "The New Weibull Handbook Fifth Edition, Reliability and Statistical Analysis for Predicting Life, Safety, Supportability, Risk, Cost and Warranty Claims." Published and distributed by Robert B. Abernethy (2006).

[5] Laidler, Keith J. "The development of the Arrhenius equation." J. Chem. Educ 61.6 (1984): 494.

[6] Ageing Assessment, Condition Inspection and Lifetime Evaluation for Safety Related Fuse in Nuclear Power Plant, J. Shi, Journal of Energy and Power Engineering 5 (2011) 892-898.

[7] Krumbein, Simeon J. "Metallic electromigration phenomena." IEEE transactions on components, hybrids, and manufacturing technology 11.1 (1988): 5-15.

[8] J.R. Black: Electromigration - A Brief Survey and Some Recent Results. IEEE Trans. on Electron Devices, Vol. ED-16 (No. 4), pp. 338-347, April 1969.

[9] Misra, E., and T. L. Alford. "Effect of alloying and cladding on the failure of silver metallization under high temperature and current stressing." Applied Physics Letters 87.17 (2005): 172111.

[10] Handbook, Military. "Reliability Prediction of Electronic Equipment (MIL-HDBK-217F)." US Government Printing Office, Washington DC (1986). 